\title{
VLT/SPHERE survey for exoplanets around young early-type stars, including systems with multi-belt architectures
}

\author{
M. Lombart ${ }^{1,2}$, G. Chauvin ${ }^{1,3}$, P. Rojo ${ }^{4}$, E. Lagadec ${ }^{5}$, P. Delorme ${ }^{3}$, H. Beust ${ }^{3}$, M. Bonnefoy ${ }^{3}$, R. Galicher ${ }^{6}$,
} R. Gratton ${ }^{7}$, D. Mesa ${ }^{7}$, M. Bonavita ${ }^{8}$, F. Allard ${ }^{9}$, A. Bayo ${ }^{10,11}$, A. Boccaletti ${ }^{6}$, S. Desidera ${ }^{7}$, J. Girard ${ }^{12}$, J. S. Jenkins ${ }^{4}$, H. Klahr ${ }^{15}$, G. Laibe ${ }^{2}$, A.-M. Lagrange ${ }^{3}$, C. Lazzoni ${ }^{7}$, G-D. Marleau ${ }^{13,14,15}$, D. Minniti ${ }^{16}$, and C. Mordasini ${ }^{14}$

${ }^{1}$ Unidad Mixta Internacional Franco-Chilena de Astronomía, CNRS/INSU UMI 3386 and Departamento de Astronomía,

Universidad de Chile, Casilla 36-D, Santiago, Chile

${ }^{2}$ Univ. Lyon, Univ. Lyon1, ENS de Lyon, CNRS, Centre de Recherche Astrophysique de Lyon UMR5574, 69230 Saint-Genis-Laval, France

e-mail: maxime. lombart@ens-lyon.fr

${ }^{3}$ Univ. Grenoble Alpes, CNRS, IPAG, 38000 Grenoble, France

${ }^{4}$ Departamento de Astronomía, Universidad de Chile, Camino El Observatorio 1515, Las Condes, Santiago, Chile

${ }^{5}$ Université Cote d'Azur, OCA, CNRS, Lagrange, France

${ }^{6}$ LESIA, Observatoire de Paris, PSL Research University, CNRS, Sorbonne Universités, UPMC Univ. Paris 06, Univ. Paris Diderot, Sorbonne Paris Cité, 5 place Jules Janssen, 92195 Meudon, France

${ }^{7}$ INAF - Osservatorio Astronomico di Padova, Vicolo dell' Osservatorio 5, 35122 Padova, Italy

${ }^{8}$ SUPA, Institute for Astronomy, The University of Edinburgh, Royal Observatory, Blackford Hill, Edinburgh, EH9 3HJ, UK

${ }^{9}$ CRAL, UMR 5574, CNRS, Université de Lyon, Ecole Normale Suprieure de Lyon, 46 allée d'Italie, 69364 Lyon Cedex 07, France

${ }^{10}$ Instituto de Física y Astronomía, Facultad de Ciencias, Universidad de Valparaíso, Av. Gran Bretaña 1111, Valparaíso, Chile

${ }^{11}$ Núcleo Milenio Formación Planetaria - NPF, Universidad de Valparaíso, Valparaíso, Chile

12 Space Telescope Science Institute, 3700 San Martin Dr. Baltimore, MD 21218, USA

13 Institut für Astronomie und Astrophysik, Universität Tübingen, Auf der Morgenstelle 10, 72076 Tübingen, Germany

${ }^{14}$ Physikalisches Institut, Universität Bern, Gesellschaftsstr. 6, 3012 Bern, Switzerland

15 Max-Planck-Institut für Astronomie, Königstuhl 17, 69117 Heidelberg, Germany

${ }^{16}$ Departamento de Ciencias Fisicas, Facultad de Ciencias Exactas, Universidad Andres Bello, Av. Fernandez Concha 700 , Las Condes, Santiago, Chile

Received 13 March 2020 / Accepted 13 May 2020

\begin{abstract}
Context. Dusty debris disks around pre- and main-sequence stars are potential signposts for the existence of planetesimals and exoplanets. Giant planet formation is therefore expected to play a key role in the evolution of the disk. This is indirectly confirmed by extant submillimeter near-infrared images of young protoplanetary and cool dusty debris disks around main-sequence stars that usually show substantial spatial structures. With two decades of direct imaging of exoplanets already studied, it is striking to note that a majority of recent discoveries of imaged giant planets have been obtained around young early-type stars hosting a circumstellar disk.

Aims. Our aim was to create a direct imaging program designed to maximize our chances of giant planet discovery and target 22 young early-type stars. About half of them show indications of multi-belt architectures.

Methods. Using the IRDIS dual-band imager and the IFS integral field spectrograph of SPHERE to acquire high-constrast coronagraphic differential near-infrared images, we conducted a systematic search in the close environment of these young, dusty, and early-type stars. We used a combination of angular and spectral differential imaging to reach the best detection performances down to the planetary mass regime.

Results. We confirm that companions detected around HIP 34276, HIP 101800, and HIP 117452 are stationary background sources and binary companions. The companion candidates around HIP 8832, HIP 16095, and HIP 95619 are determined as background contaminations. Regarding the stars for which we infer the presence of debris belts, a theoretical minimum mass for planets required to clear the debris gaps can be calculated. The dynamical mass limit is at least $0.1 M_{\mathrm{J}}$ and can exceed $1 M_{\mathrm{J}}$. Direct imaging data is typically sensitive to planets down to $\sim 3.6 M_{\mathrm{J}}$ at $1^{\prime \prime}$, and $1.7 M_{\mathrm{J}}$ in the best case. These two limits tightly constrain the possible planetary systems present around each target. These systems will be probably detectable with the next generation of planet imagers.
\end{abstract}

Key words. techniques: imaging spectroscopy - planets and satellites: detection - planets and satellites: fundamental parameters planets and satellites: atmospheres

\section{Introduction}

How giant planets form and evolve is one of the biggest challenges of modern astronomy and remains a subject of heated debate. This major goal is directly connected to the ultimate search for life over the horizon 2030 to 2040, although several astrophysical (formation, evolution, dynamics, structure, and atmosphere), biological (bio-markers), and technical (new technologies developed for next generation of instrumentation) steps must be carried out in that perspective. Understanding how giant planets are formed and structured, how they evolve and interact, is critical as they completely shape the planetary system 
architectures and therefore the possibility of forming telluric planets capable of hosting life. More than two decades ago, the only planets we knew were the ones of our Solar System. With the manna of exoplanet discoveries since the 51 Peg discovery (Mayor et al. 1995), the diversities of systems found (hot Jupiters, irradiated and evaporating planets, misaligned planets with stellar spin, planets in binaries, telluric planets in habitable zones, discovery of Mars-sized planets...), the theories of planetary formation have drastically evolved to digest these observing constraints. However, we are still missing the full picture, and some key fundamental questions still lack answers. For example: (i) the physical processes at play to pass the $\mathrm{km}$-size barrier to form planetary cores, (ii) the physics of accretion to form planetary atmospheres, (iii) the formation mechanisms to explain the existence of giant planets at wide orbits, (iv) the physical properties of young Jupiters, (v) the impact of planet-planet and planet-disk interaction in the final planetary system architecture, or (vi) the influence of the stellar mass and stellar environment in the planetary formation processes. Neither core accretion plus gas capture (CA; Pollack et al. 1996) nor disk fragmentation driven by gravitational instabilities (GI; Cameron 1978) can globally explain all current observables from planet hunting techniques. Alternative mechanisms are then proposed, such as pebbles accretion to enable core accretion to operate at wide orbits (Lambrechts \& Johansen 2012), inward/outward migration or planet-planet (Crida et al. 2009; Bromley \& Kenyon 2014) or simply the possibility to have several mechanisms forming giant planets (Boley 2009). In this context, each individual discovery of a giant planet and young planetary system using direct imaging is rich in terms of scientific exploitation and characterization, as these systems offer the possibility of (i) directly probing the presence of planets in their birth environments, (ii) enabling the orbital, physical, and spectral characterization of young massive Jupiters, (iii) characterizing the population of giant planets at all separations in synergy with complementary techniques such as astrometry (Gaia) and radial velocity adapted to filter stellar activity.

Dusty debris disks around pre- and main-sequence stars are possible signposts for the existence of planetesimals and exoplanets (Matthews et al. 2014). Numerous T Tauri and Herbig stars indicate that the characteristic timescale for the dispersal of a surrounding dusty, gaseous disk is a few million years (Kennedy \& Kenyon 2008). Giant planet formation is therefore expected to play a key role in the evolution of disk. This is indirectly confirmed by extant submillimeter and near-infrared images of cool dusty debris disks around main-sequence stars usually showing substantial spatial structure (e.g., $\epsilon$ Eri, Vega, Fomalhaut, $\beta$ Pic; see Schneider et al. 2014). It is striking to note that a majority of recent discoveries of imaged giant planets have been obtained around young, dusty, early-type stars. It includes the breakthrough discoveries of Fomalhaut b ( $3 M_{\text {Jup }}$ at $110 \mathrm{AU}$, A4V star; Kalas et al. 2008), HR 8799 bcde (5-10 $M_{\text {Jup }}$ at 10$64 \mathrm{au}, \mathrm{F0V}$ star; Marois et al. 2010), $\beta$ Pictoris b (8-13 $M_{\text {Jup }}$ at 9 au, A5V star; Lagrange et al. 2010), HD 95086 b (3-5 $M_{\text {Jup }}$ at 56 au, A8V star; Rameau et al. 2013), and more recently 51 Eri b (2 $M_{\text {Jup }}$ at $14 \mathrm{au}$, F0V star; Macintosh et al. 2015). The presence of dust and the spatial substructure (ring, gap, warp, and other asymmetries) are possible indirect indicators of the presence of giant planets (Mouillet et al. 1997; Dipierro et al. 2015; Pinte et al. 2020). Direct imaging is here a unique and viable technique to complete our view of planetary system characteristics at wide orbits ( $\geq 5 \mathrm{au}$ ). This technique enables us to directly study the planet-disk connection to constrain the planet's and disk's physical properties, evolution, and formation. In the case of $\beta$ Pictoris, Lagrange et al. (2012) confirmed that $\beta$ Pic $\mathrm{b}$ was actually responsible for the disk inner warp geometry, perturbing the planetesimals field and shaping the warp up to 40-60 au. The stars HD 95086 and HR 8799 share a common two-component architecture consisting of a warm inner belt ( $\leq 5 \mathrm{au})$ and a cold outer disk (100-200 au) (see Su et al. 2015). Kennedy \& Wyatt (2014) actually showed that the spectral energy distributions of both systems are consistent with two-temperature components compatible with dust emission arising from two distinct radial locations. Such an architecture would be analogous to the outer Solar System's configuration of asteroid and Kuiper belts separated by giant planets. Therefore, following the strategy of our NaCo DUSTIES (Dusty, yoUng, and early-type STar Imaging for ExoplanetS) survey (Rameau et al. 2013) that led to the discovery of HD $95086 \mathrm{~b}$, we initiated a searching for giant planets with SPHERE at VLT around an newly identified sample of young early-type stars with indication for some cases of multibelt architecture to maximize the chances of discoveries. The sample, the observations, and the data reduction and analysis are presented in Sects. 2-4, respectively. The results are reported in Sect. 5 and discussed in Sect. 6.

\section{Target properties}

The target selection of the survey was obtained from a large sample of young, nearby early-type stars according to the following criteria: declination $\left(\delta \leq 25^{\circ}\right)$, age $(\leq 100 \mathrm{Myr})$, distance ( $\leq 100 \mathrm{pc}$ ), and $R$-band brightness $(\leq 9.5)$ to favor good adaptive optics performances. Age selection criteria were applied based on different youth diagnostics (kinematics, isochrones, lithium, $\mathrm{H}_{\alpha}$ emission, X-ray activity, stellar rotation, and chromospheric activity). We also used, as selection criteria, the presence of significant $60-70 \mu \mathrm{m}$ excess from the IRAS and Spitzer missions in the spectral energy distributions (Zuckerman et al. 1995, 2011, 2013; Zuckerman 2001; Rhee et al. 2007; Zuckerman \& Song 2004a,b; David \& Hillenbrand 2015; Moór et al. 2016) or the existence of multi-belt component analysis from Kennedy \& Wyatt (2014). A final total of 30 late-B-, A-, and early-Ftype young stars, observable from the southern hemisphere, were then kept, 22 of which were observed between October 2016 and August 2019. Their stellar properties are reported in Table 1. The age, distance, spectral type, and IR excess properties are shown in Fig. 1.

\section{Observations}

The SPHERE planet-finder instrument installed at the VLT (Beuzit et al. 2019) is a highly specialized instrument, dedicated to high-contrast imaging and spectroscopy of young giant exoplanets. It is based on the SAXO extreme adaptive optics (XAO) system (Fusco et al. 2006; Sauvage et al. 2010; Petit et al. 2014), which controls a deformable mirror with $41 \times$ 41 actuators, and four control loops (fast visible tip-tilt, highorders, near-infrared differential tip-tilt, and pupil stabilization). The common path optics employ several stress-polished toric mirrors (Hugot et al. 2012) to transport the beam to the coronagraphs and scientific instruments. Several types of coronagraphic devices for stellar diffraction suppression are provided, including apodized pupil Lyot coronagraphs (Soummer et al. 2005) and achromatic four-quadrant phase masks (Boccaletti et al. 2008). The instrument has three science subsystems: the infrared dual-band imager and spectrograph (IRDIS, Dohlen et al. 2008), an integral field spectrograph (IFS; Claudi et al. 2008), 
Table 1. Description and properties of the sample.

\begin{tabular}{|c|c|c|c|c|c|c|c|c|c|c|}
\hline Target & $\operatorname{RA}(2000)$ & $\operatorname{Dec}(2000)$ & $\begin{array}{l}\mu_{\alpha} \\
\left(\operatorname{mas~yr}^{-1}\right)\end{array}$ & $\begin{array}{l}\mu_{\delta} \cdot \cos (\delta) \\
\left(\operatorname{mas~yr}^{-1}\right)\end{array}$ & $\begin{array}{l}H \\
\text { (mag) }\end{array}$ & SpT & $\begin{array}{l}\text { Dist. } \\
\text { (pc) }\end{array}$ & $\begin{array}{l}\text { Age } \\
(\mathrm{Myr})\end{array}$ & Exc. & References \\
\hline HIP 3277 & 004146.3 & -563004.73 & 90.79 & 57.19 & 5.6 & A3V & 67 & $93_{-76}^{+283}$ & I & D15 \\
\hline HIP 7345 & 013437.7 & -154034.89 & 94.84 & -3.14 & 5.5 & A1V & 61 & $35_{-5}^{+5}$ & $\mathrm{Y}$ & Z95; Z12; G16 \\
\hline HIP 7805 & 014024.0 & -605953.62 & 61.94 & -10.50 & 6.7 & F2V & 66 & $30_{-15}^{+15}$ & $\mathrm{Y}$ & Z01; Z04; M17 \\
\hline HIP 8832 & 015331.8 & +19 1737.87 & 79.20 & -97.63 & 2.8 & A0 & 50 & $87_{-71}^{+195}$ & / & D15 \\
\hline HIP 9902 & 020726.1 & -594045.942 & 91.11 & -18.29 & 6.2 & F7V & 44 & $45_{-4}^{+4}$ & $\mathrm{Y}$ & K14; B15 \\
\hline HIP 13141 & 024901.4 & -624823.47 & 94.02 & 29.10 & 5.2 & $\mathrm{~A} 2 \mathrm{~V}$ & 50 & $100_{-70}^{+200}$ & $\mathrm{Y}$ & R07; G16 \\
\hline HIP 16095 & 032718.6 & +124407.03 & 10.36 & -7.56 & 6.3 & $\mathrm{~A} 0 \mathrm{~V}$ & 88 & $194_{-138}^{+171}$ & / & Z13; D15 \\
\hline HIP 18437 & 035629.3 & -385743.80 & 29.46 & 0.10 & 6.8 & A0V & 100 & $187_{-177}^{+150}$ & $\mathrm{Y}$ & R07; M17 \\
\hline HIP 19990 & 041715.6 & +203442.93 & -39.41 & -60.79 & 4.6 & A3 & 29 & $70_{-40}^{+30}$ & / & Z13; G16 \\
\hline HIP 22192 & 044625.7 & -280514.8 & -3.82 & 17.58 & 5.7 & A3V & 56 & $12_{-5}^{+5}$ & / & Z13; G16 \\
\hline HIP 22226 & 044649.5 & -261808.84 & 34.52 & -4.13 & 6.9 & F3V & 78 & $30_{-20}^{+20}$ & $\mathrm{Y}$ & R07; G16 \\
\hline HIP 22845 & 045453.7 & +100902.99 & 41.49 & -128.73 & 4.5 & A3V & 34 & $100_{-70}^{+200}$ & $\mathrm{Y}$ & Z04b; G16 \\
\hline HIP 26309 & 053610.2 & -284228.847 & 25.80 & -3.04 & 5.9 & $\mathrm{~A} 2 \mathrm{~V}$ & 56 & $30_{-10}^{+20}$ & / & Z11; G16 \\
\hline HIP 26990 & 054335.8 & -395524.7145 & 25.82 & 15.08 & 6.8 & G0V & 55 & $42_{-7}^{+8}$ & $\mathrm{Y}$ & M16; V17 \\
\hline HIP 34276 & 070620.9 & -433638.69 & 5.80 & 13.20 & 6.5 & $\mathrm{~A} 0 \mathrm{~V}$ & 102 & $185_{-170}^{+120}$ & $\mathrm{Y}$ & R07; M17 \\
\hline HIP 41307 & 082539.6 & -035423.11 & -66.43 & -23.41 & 3.9 & A0V & 37 & $203_{-100}^{+100}$ & $\mathrm{Y}$ & R07; M17 \\
\hline HIP 93542 & 190306.8 & -420542.38 & 56.41 & -46.43 & 5.0 & B9V & 59 & $76_{-62}^{+148}$ & $\mathrm{Y}$ & R07; D15 \\
\hline HIP 95619 & 192656.4 & -294435.617 & 18.63 & -50.13 & 5.7 & B8.5 & 70 & $86_{-69}^{+138}$ & $\mathrm{Y}$ & D15 \\
\hline HIP 97749 & 195150.6 & -395227.7 & 18.42 & -11.27 & 5.4 & A & 100 & $82_{-67}^{+177}$ & / & D15 \\
\hline HIP 101800 & 203749.1 & +112239.63 & 39.15 & -8.26 & 5.4 & A1V & 57 & $225_{-43}^{+311}$ & $\mathrm{Y}$ & R07; D15 \\
\hline HIP 101958 & 203938.2 & +155443.46 & 53.82 & 8.47 & 3.9 & B9V & 77 & $60_{-49}^{+164}$ & I & D15 \\
\hline HIP 117452 & 234855.5 & -280748.97 & 100.80 & -105.34 & 4.6 & A0V & 42 & $70_{-40}^{+30}$ & $\mathrm{Y}$ & Z11; D15 \\
\hline
\end{tabular}

Notes. The Exc. column indicates the presence of an IR excess. The symbol " " means no IR excess, and "Y" means with IR excess.

References. (B15) Bell et al. (2015); (D15) David \& Hillenbrand (2015); (G16) Galicher et al. (2016); (K14) Kennedy \& Wyatt (2014); (M16) Moór et al. (2016); (M17) Meshkat et al. (2017); (R07) Rhee et al. (2007); (V17) Vigan et al. (2017); (Z95) Zuckerman et al. (1995); (Z01) Zuckerman (2001); (Z04) Zuckerman \& Song (2004a); (Z04b) Zuckerman \& Song (2004b); (Z11) Zuckerman et al. (2011); (Z12) Zuckerman \& Song (2012); (Z13) Zuckerman et al. (2013).

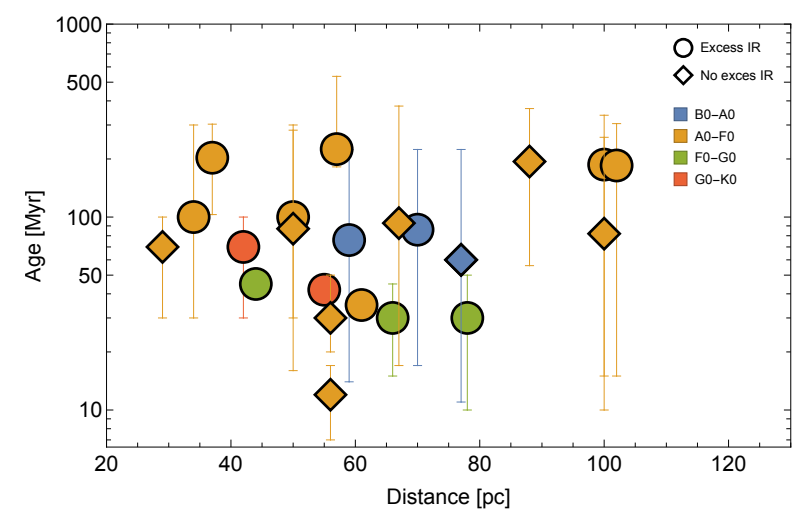

Fig. 1. Diagram of target properties taking into account age with error bars, distance, spectral type, and excess in infrared.

and the Zimpol rapid-switching imaging polarimeter (ZIMPOL; Thalmann et al. 2008).

The sample of young early-type stars was observed using the IRDIFS-EXT mode, with IRDIS in the dual-band imaging
(DBI, Vigan et al. 2010) mode with $K_{1} K_{2}$ filters $\left(\lambda_{K_{1}}=\right.$ $\left.2.1025 \pm 0.1020 \mu \mathrm{m}, \lambda_{K_{2}}=2.2550 \pm 0.1090 \mu \mathrm{m}\right)$, and IFS in the $Y-H(0.97-1.66 \mu \mathrm{m})$ mode in pupil-tracking. This combination enables the use of angular and/or spectral differential imaging techniques to improve the contrast performances at the subarcsecond level (Racine et al. 1999; Marois et al. 2006). The choice between IRDIFS mode and IRDIFS-EXT mode is critical to optimizing the detection of young, early-T, or warm, mid-L dwarfs planets, considering the primary age and distance. Indeed, it was crucial in the cases of the $\beta$ Pic b (Lagrange et al. 2009) and HD 95086 b (Rameau et al. 2013) discoveries to properly remove quasi-static speckles that dominate performance detection at close inner angles $\left(0.1-2.0^{\prime \prime}\right.$, i.e., 3-60 au at $30 \mathrm{pc}$ ), but to also maximize the emitted flux by the giant planets. For young ages $(10-50 \mathrm{Myr})$, as the potential planets to which we are mostly sensitive are warm and dusty L-type planets with no methane absorption, the choice of the IRDIFS-EXT mode is more appropriate and was chosen for this observing campaign. For the follow-up, as candidates were only detected in the IRDIS field of view, we opted for the use of the IRDIS the DBI mode with $J_{2} J_{3}$ filters $\left(\lambda_{J_{2}}=2.1025 \pm 0.1020 \mu \mathrm{m}\right.$, 
$\left.\lambda_{J_{3}}=2.2550 \pm 0.1090 \mu \mathrm{m}\right)$ in pupil-tracking. Thus, this second epoch provides, in addition to the possibility of checking for common proper motion of the candidates relative to the primary star, the possibility to better discriminate background stars from physically young, early-T, or warm mid-L dwarfs planets in the color-magnitude diagram (Bonnefoy et al. 2018).

The observing sequence used for the survey is as follows; PSF flux reference, coronographic centering using the waffle spots, deep coronographic observation of about $70 \mathrm{~min}$ in total on target, new coronographic centering using the waffle spots, PSF flux reference, and sky. The PSF flux references were used to estimate the relative photometry of the companion candidates detected in the IRDIS and IFS field of view, as well as the detection limits. The coronographic centering sequence using the waffle spots sequence is critical to obtaining the position of the star behind the coronograph and the relative astrometry of the companion candidates. The deep coronographic observation was obtained close to meridian to maximize the field rotation. Finally, the sky background was used to optimize the background subtraction and the flat field correction. The typical observing sequence lasts approximately $90 \mathrm{~min}$, including pointing and overheads. The detail of the observations per target is reported in Table 2. As a by-product of the SPHERE observation, one can access the evolution of the different atmospheric parameters seen and registered by the SPHERE XAO system (SAXO). These real-time parameters are good diagnostics of the turbulence conditions $\left(\tau_{0}, r_{0}\right.$, integrated wind over the line of sight) and of the XAO correction (Strehl at $1.6 \mu \mathrm{m}$ ) during the observing sequence. The summary of these SAXO parameters over the full survey is reported in Table 2 and shown in Fig. 2. Given the brightness of our targets, about $70 \%$ of the survey was obtained under median or good conditions for Paranal, with a typical Strehl ratio larger than $80 \%$. Prior to the UT3 intervention at VLT in 2017, a few cases were affected by the low-wind effect, despite good atmospheric conditions.

\section{Data reduction and analysis}

In order to calibrate the IRDIS and IFS dataset on sky, the platescale and true north solution at each epoch were corrected based on the long-term analysis of the SPHERE Guaranteed Time Observation astrometric calibration described by Maire et al. (2016). The rotation correction considered to align images to the detector vertical in pupil-tracking observations is $-135.99 \pm 0.11^{\circ}$. Anamorphism correction was obtained by stretching the image $Y$-direction with a factor of $1.0060 \pm$ 0.0002. All IRDIS and IFS datasets were reduced using the SPHERE Data Reduction and Handling (DRH) automated pipeline (Pavlov et al. 2008) and additional IDL routines for the IFS data reduction (Mesa et al. 2015) at the SPHERE Data Center (Delorme et al. 2017) to correct each data cube for bad pixels, dark current, flat field, and sky background. After combining all data cubes with an adequate calculation of the parallactic angle for each individual frame of the deep coronagraphic sequence, all frames were shifted at the position of the stellar centroid calculated from the initial star center position.

For an independent check, two pipelines were then used to process the data in angular differential imaging (ADI), and in combined spectral and angular differential imaging (ASDI): the IPAG-ADI pipeline (Chauvin et al. 2012), and the SpeCal (Galicher et al. 2018). These routines allowed us to reduce the data cubes with almost the same set of algorithms (classical ADI, Marois et al. 2006; LOCI, Lafrenière et al. 2007; PCA,
Soummer et al. 2012; Andromeda, Cantalloube et al. 2015), and to exploit the spectral diversity of the IRDIS and IFS observations using ASDI techniques in addition to ADI only. Following the principles described in Galicher et al. (2018), SpeCal (and IPAG-ADI) delivers, for various algorithms and observing techniques (ADI, ASDI), contrast curves, signal-to-noise ratio ( $/ \mathrm{N}$ ) maps, and the possibility to locally characterize the astrometric, photometric, and spectroscopic signal of any companion candidate using either a template or negative fake planet injection approach. As consistent results were found with both pipelines, the full set of observations was reduced with SpeCal (routinely used with the SPHERE GTO) using the TLOCI algorithm (in ADI and ASDI) for IRDIS, and the PCA algorithm (in ASDI) for IFS. A spatial filtering for each data cube was automatically applied to the deep coronographic observations and the reference PSFs before the use of SpeCal.

The TLOCI algorithm is implemented in SpeCal, to attenuate the background signal. The TLOCI algorithm locally subtracts the stellar speckle pattern for each frame in annuli of $1.5 \times F W H M$ further divided into sectors. The subtraction is based on a linear combination of the best 20 ( $N$ parameter) correlated reference images calculated in the optimization region and selected to minimize the self-subtraction at a maximum of $20 \%$ ( $\tau$ parameter), see Galicher et al. (2011) and Marois et al. (2014) for a further description of the reference frame selection and the subtraction and optimization regions. For IFS, in the PCA version, each frame we used is subtracted from its average over the field of view to estimate the principal components. The spectral diversity is exploited after proper rescaling and renormalization of the IFS data cubes as detailed by Mesa et al. (2015). Considering the significant field rotation of our observations, the first 100 principal components were subtracted.

\section{Companion candidate detection and characterization}

Using the IRDIS and IFS S/N maps provided by SpeCal, we identified a total of eight companion candidates by eye at relatively large separation $\left(\geq 3.0^{\prime \prime}\right)$ in the IRDIS fields of view of six targets (HIP 16095, HIP 95619, HIP 101800, HIP 34276 , HIP 117452, and HIP 8832) of the complete survey. One companion candidate was identified at relatively close separation in the IFS field of view of HIP 41307, but later flagged as a bright quasi-static speckle through various processing tests, and was therefore discarded.

Figure 3 shows the IRDIS image reduced in TLOCI ADI of HIP 16095 (bright companion located at 3.3" in the $K_{1}$ and $K_{2}$ combined filters), and the IFS image reduced in PCA ASDI of HIP 41307 (quasi-static speckle discarded located at $0.5^{\prime \prime}$ in the combined $Y J H$-bands) as an illustration of the detection process. All companion candidates were then characterized using SpeCal with the TLOCI algorithm in ADI only, and according to a template approach. The relative astrometry and photometry are reported in Table 3. As first diagnostics, in Fig. 4 (left), we reported the location of all our companion candidates in the $K_{1}$-band- and $K_{2}$-band-based color-magnitude diagram (CMD). Details on the diagrams are given in Mesa et al. (2016), Samland et al. (2017), Chauvin et al. (2018), and Bonnefoy et al. (2018). We used the most recent parallaxes of the young objects from Greco \& Brandt (2016), and added additional companions (Gauza et al. 2015; Stone et al. 2016; De Rosa et al. 2014) at the L/T transition. At first glance, we see that all detected companion candidates fall on the expected sequence of possible 
Table 2. Observing log.

\begin{tabular}{|c|c|c|c|c|c|c|c|c|c|c|}
\hline UT date & Target & Instrument & Mode & Filter & $\begin{array}{l}\text { NDIT } \times \text { DIT } \\
(s)\end{array}$ & $N_{\text {exp }}$ & $\begin{array}{l}\Delta \theta \\
\left(^{\circ}\right)\end{array}$ & $\begin{array}{l}\omega \\
\left({ }^{\prime \prime}\right)\end{array}$ & $\begin{array}{l}\text { Strehl } \\
\text { at } 1.6 \mu \mathrm{m}\end{array}$ & Airmass \\
\hline \multicolumn{11}{|c|}{ Survey } \\
\hline \multirow{2}{*}{$05-10-2016$} & HIP 9902 & $\begin{array}{l}\text { IRDIS } \\
\text { IFS }\end{array}$ & $\begin{array}{l}\mathrm{DBI} \\
R_{\lambda}=30\end{array}$ & $\begin{array}{l}K_{1} K_{2} \\
\text { YJH }\end{array}$ & $\begin{array}{l}3 \times 64 \\
1 \times 64\end{array}$ & 46 & 20.7 & 0.62 & 0.75 & 1.22 \\
\hline & HIP 18437 & $\begin{array}{l}\text { IRDIS } \\
\text { IFS }\end{array}$ & $\begin{array}{l}\text { DBI } \\
R_{\lambda}=30\end{array}$ & $\begin{array}{l}K_{1} K_{2} \\
\text { YJH }\end{array}$ & $\begin{array}{l}3 \times 64 \\
1 \times 64\end{array}$ & 46 & 44.2 & 0.47 & 0.77 & 1.03 \\
\hline \multirow{2}{*}{ 07-10-2016 } & HIP 7805 & $\begin{array}{l}\text { IRDIS } \\
\text { IFS }\end{array}$ & $\begin{array}{l}\mathrm{DBI} \\
R_{\lambda}=30\end{array}$ & $\begin{array}{l}K_{1} K_{2} \\
\text { YJH }\end{array}$ & $\begin{array}{l}3 \times 64 \\
1 \times 64\end{array}$ & 46 & 20.0 & 0.53 & 0.83 & 1.24 \\
\hline & HIP 16095 & $\begin{array}{l}\text { IRDIS } \\
\text { IFS }\end{array}$ & $\begin{array}{l}\text { DBI } \\
R_{\lambda}=30\end{array}$ & $\begin{array}{l}K_{1} K_{2} \\
\text { YJH }\end{array}$ & $\begin{array}{l}3 \times 64 \\
1 \times 64\end{array}$ & 46 & 19.0 & 0.46 & 0.87 & 1.26 \\
\hline 08-10-2016 & HIP 13141 & $\begin{array}{l}\text { IRDIS } \\
\text { IFS }\end{array}$ & $\begin{array}{l}\mathrm{DBI} \\
R_{\lambda}=30\end{array}$ & $\begin{array}{l}K_{1} K_{2} \\
\text { YJH }\end{array}$ & $\begin{array}{l}3 \times 64 \\
1 \times 64\end{array}$ & 46 & 20.8 & 0.41 & 0.83 & 1.30 \\
\hline 10-11-2016 & HIP 19990 & $\begin{array}{l}\text { IRDIS } \\
\text { IFS }\end{array}$ & $\begin{array}{l}\mathrm{DBI} \\
R_{\lambda}=30\end{array}$ & $\begin{array}{l}K_{1} K_{2} \\
\text { YJH }\end{array}$ & $\begin{array}{l}3 \times 64 \\
1 \times 32\end{array}$ & 46 & 22.6 & 0.27 & 0.94 & 1.30 \\
\hline $12-11-2016$ & HIP 26309 & $\begin{array}{l}\text { IRDIS } \\
\text { IFS }\end{array}$ & $\begin{array}{l}\mathrm{DBI} \\
R_{\lambda}=30\end{array}$ & $\begin{array}{l}K_{1} K_{2} \\
\text { YJH }\end{array}$ & $\begin{array}{l}3 \times 64 \\
1 \times 64\end{array}$ & 46 & 107.4 & 0.41 & 0.87 & 1.01 \\
\hline 13-11-2016 & HIP 22192 & $\begin{array}{l}\text { IRDIS } \\
\text { IFS }\end{array}$ & $\begin{array}{l}\mathrm{DBI} \\
R_{\lambda}=30\end{array}$ & $\begin{array}{l}K_{1} K_{2} \\
\text { YJH }\end{array}$ & $\begin{array}{l}7 \times 32 \\
1 \times 32\end{array}$ & 46 & 130.9 & 0.33 & 0.86 & 1.01 \\
\hline 04-12-2016 & HIP 7345 & $\begin{array}{l}\text { IRDIS } \\
\text { IFS }\end{array}$ & $\begin{array}{l}\mathrm{DBI} \\
R_{\lambda}=30\end{array}$ & $\begin{array}{l}K_{1} K_{2} \\
\text { YJH }\end{array}$ & $\begin{array}{l}3 \times 64 \\
1 \times 64\end{array}$ & 17 & 81.4 & 0.44 & 0.90 & 1.02 \\
\hline $05-12-2016$ & HIP 22226 & $\begin{array}{l}\text { IRDIS } \\
\text { IFS }\end{array}$ & $\begin{array}{l}\mathrm{DBI} \\
R_{\lambda}=30\end{array}$ & $\begin{array}{l}K_{1} K_{2} \\
\text { YJH }\end{array}$ & $\begin{array}{l}3 \times 64 \\
1 \times 64\end{array}$ & 46 & 15.2 & 0.42 & 0.82 & 1.00 \\
\hline $07-12-2016$ & HIP 22845 & $\begin{array}{l}\text { IRDIS } \\
\text { IFS }\end{array}$ & $\begin{array}{l}\text { DBI } \\
R_{\lambda}=30\end{array}$ & $\begin{array}{l}K_{1} K_{2} \\
\text { YJH }\end{array}$ & $\begin{array}{l}3 \times 64 \\
1 \times 32\end{array}$ & 46 & 19.3 & 0.44 & 0.82 & 1.27 \\
\hline $13-12-2016$ & HIP 34276 & $\begin{array}{l}\text { IRDIS } \\
\text { IFS }\end{array}$ & $\begin{array}{l}\text { DBI } \\
R_{\lambda}=30\end{array}$ & $\begin{array}{l}K_{1} K_{2} \\
\text { YJH }\end{array}$ & $\begin{array}{l}8 \times 32 \\
1 \times 64\end{array}$ & 46 & 39.5 & 0.55 & 0.84 & 1.06 \\
\hline $15-12-2016$ & HIP 26990 & $\begin{array}{l}\text { IRDIS } \\
\text { IFS }\end{array}$ & $\begin{array}{l}\text { DBI } \\
R_{\lambda}=30\end{array}$ & $\begin{array}{l}K_{1} K_{2} \\
\text { YJH }\end{array}$ & $\begin{array}{l}3 \times 64 \\
1 \times 64\end{array}$ & 46 & 42.6 & 0.55 & 0.76 & 1.04 \\
\hline $15-12-2010$ & HIP 41307 & $\begin{array}{l}\text { IRDIS } \\
\text { IFS }\end{array}$ & $\begin{array}{l}\mathrm{DBI} \\
R_{\lambda}=30\end{array}$ & $\begin{array}{l}K_{1} K_{2} \\
\text { YJH }\end{array}$ & $\begin{array}{l}17 \times 16 \\
1 \times 16\end{array}$ & 46 & 43.0 & 0.35 & 0.92 & 1.03 \\
\hline $1706-2017$ & HIP 93542 & $\begin{array}{l}\text { IRDIS } \\
\text { IFS }\end{array}$ & $\begin{array}{l}\mathrm{DBI} \\
R_{\lambda}=30\end{array}$ & $\begin{array}{l}K_{1} K_{2} \\
\text { YJH }\end{array}$ & $\begin{array}{l}7 \times 32 \\
1 \times 32\end{array}$ & 46 & 59.5 & 0.83 & 0.69 & 1.05 \\
\hline $17-06-2017$ & HIP 97749 & $\begin{array}{l}\text { IRDIS } \\
\text { IFS }\end{array}$ & $\begin{array}{l}\text { DBI } \\
R_{\lambda}=30\end{array}$ & $\begin{array}{l}K_{1} K_{2} \\
\text { YJH }\end{array}$ & $\begin{array}{l}7 \times 32 \\
1 \times 32\end{array}$ & 46 & 43.3 & 0.81 & 0.52 & 1.06 \\
\hline 06-07-2017 & HIP 101800 & $\begin{array}{l}\text { IRDIS } \\
\text { IFS }\end{array}$ & $\begin{array}{l}\mathrm{DBI} \\
R_{\lambda}=30\end{array}$ & $\begin{array}{l}K_{1} K_{2} \\
\text { YJH }\end{array}$ & $\begin{array}{l}7 \times 32 \\
1 \times 32\end{array}$ & 42 & 22.1 & 0.58 & 0.86 & 1.24 \\
\hline $15-07-2017$ & HIP 117452 & $\begin{array}{l}\text { IRDIS } \\
\text { IFS }\end{array}$ & $\begin{array}{l}\mathrm{DBI} \\
R_{\lambda}=30\end{array}$ & $\begin{array}{l}K_{1} K_{2} \\
\text { YJH }\end{array}$ & $\begin{array}{l}6 \times 32 \\
1 \times 32\end{array}$ & 46 & 117.1 & 0.45 & 0.87 & 1.01 \\
\hline 20-07-2017 & HIP 101958 & $\begin{array}{l}\text { IRDIS } \\
\text { IFS }\end{array}$ & $\begin{array}{l}\text { DBI } \\
R_{\lambda}=30\end{array}$ & $\begin{array}{l}K_{1} K_{2} \\
\text { YJH }\end{array}$ & $\begin{array}{l}15 \times 16 \\
1 \times 16\end{array}$ & 46 & 23.4 & 0.45 & 0.90 & 1.36 \\
\hline 31-07-2017 & HIP 95619 & $\begin{array}{l}\text { IRDIS } \\
\text { IFS }\end{array}$ & $\begin{array}{l}\text { DBI } \\
R_{\lambda}=30\end{array}$ & $\begin{array}{l}K_{1} K_{2} \\
\text { YJH }\end{array}$ & $\begin{array}{l}7 \times 32 \\
1 \times 32\end{array}$ & 46 & 110.0 & 0.77 & 0.62 & 1.01 \\
\hline 09-08-2017 & HIP 8832 & $\begin{array}{l}\text { IRDIS } \\
\text { IFS }\end{array}$ & $\begin{array}{l}\mathrm{DBI} \\
R_{\lambda}=30\end{array}$ & $\begin{array}{l}K_{1} K_{2} \\
\text { YJH }\end{array}$ & $\begin{array}{l}15 \times 16 \\
1 \times 16\end{array}$ & 46 & 22.5 & 0.35 & 0.89 & 1.40 \\
\hline 10-09-2017 & HIP 3277 & $\begin{array}{l}\text { IRDIS } \\
\text { IFS }\end{array}$ & $\begin{array}{l}\mathrm{DBI} \\
R_{\lambda}=30\end{array}$ & $\begin{array}{l}K_{1} K_{2} \\
\text { YJH }\end{array}$ & $\begin{array}{l}7 \times 32 \\
1 \times 32\end{array}$ & 46 & 26.5 & 0.54 & 0.83 & 1.20 \\
\hline & & & & Foll & w-up & & & & & \\
\hline 27-09-2018 & HIP 117452 & IRDIS & DBI & $J_{2} J_{3}$ & $6 \times 32$ & 22 & 112.7 & 0.41 & 0.88 & 1.00 \\
\hline $10-10-2018$ & HIP 8832 & IRDIS & DBI & $J_{2} J_{3}$ & $4 \times 48$ & 23 & 20.4 & 0.61 & 0.78 & 1.00 \\
\hline 22-11-2018 & HIP 34276 & IRDIS & DBI & $J_{2} J_{3}$ & $4 \times 64$ & 23 & 46.3 & 0.39 & 0.82 & 1.44 \\
\hline 09-05-2019 & HIP 95619 & IRDIS & DBI & $J_{2} J_{3}$ & $7 \times 32$ & 23 & 22.3 & 0.51 & 0.75 & 1.02 \\
\hline 18-06-2019 & HIP 101800 & IRDIS & DBI & $J_{2} J_{3}$ & $7 \times 32$ & 23 & 20.2 & 0.68 & 0.83 & 1.36 \\
\hline
\end{tabular}

bound companions from the early-M spectral type for the candidates around HIP 117452, late-L spectral types for HIP 95619, HIP 16095 and HIP 8832, to early-T for HIP 34276. The companion around HIP 101800 was detected only in $K_{1}$-band during the first epoch. After a verification of the public archive, the companion candidates around HIP 34276 (cc1 and cc2) and HIP 101800 (cc1 and cc2) were previously known and characterized as stationary background sources by Wahhaj et al. (2013) as part of 

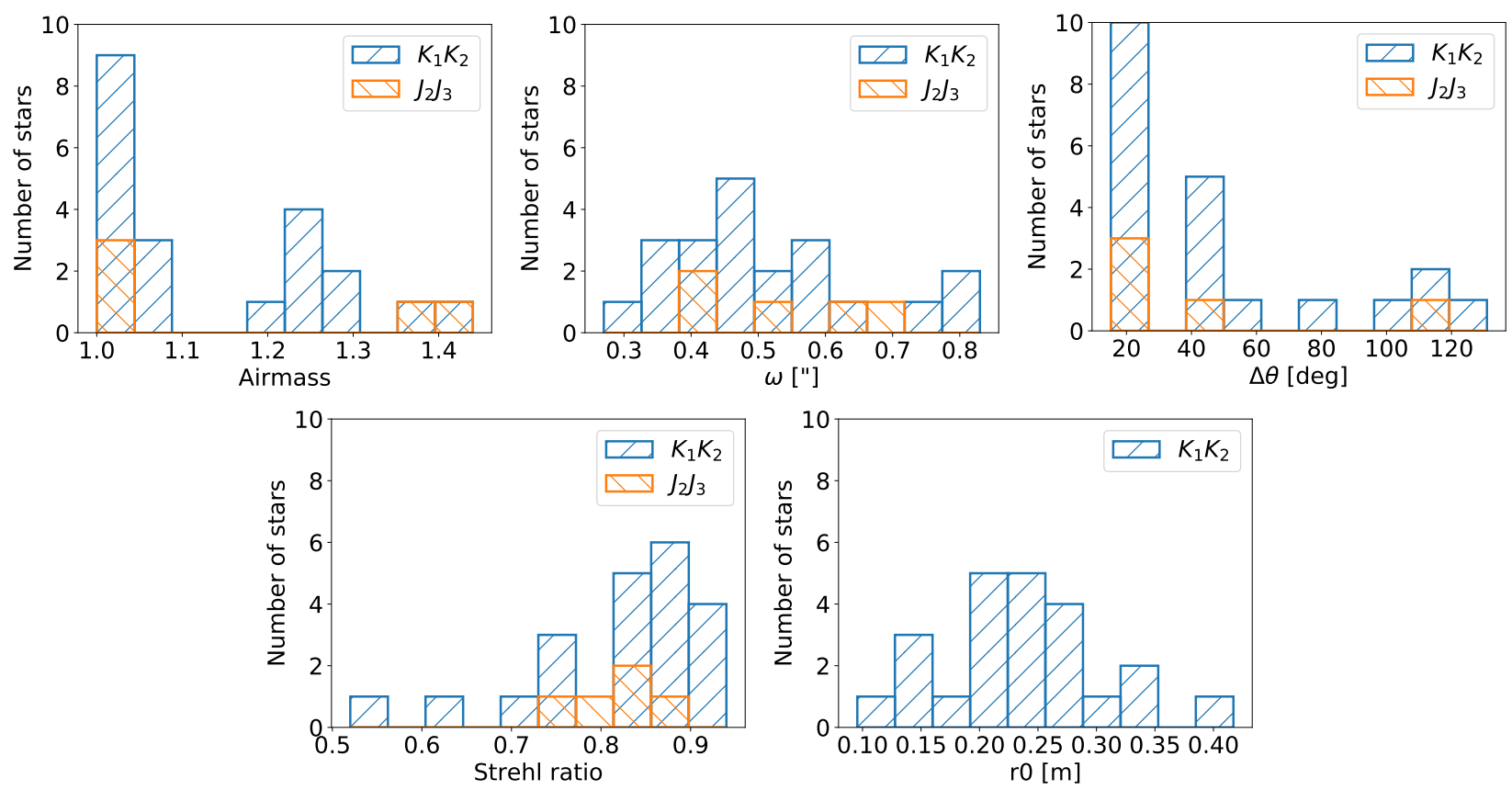

Fig. 2. Distribution of the SAXO real-time parameters, averaged over each observing sequence, for the complete survey: airmass, DIMM seeing $(\omega)$, parallactic angle variation $(\Delta \theta)$, the Strehl ratio at $1.6 \mu \mathrm{m}$, and the Fried parameter of the atmosphere $\left(r_{0}\right)$.
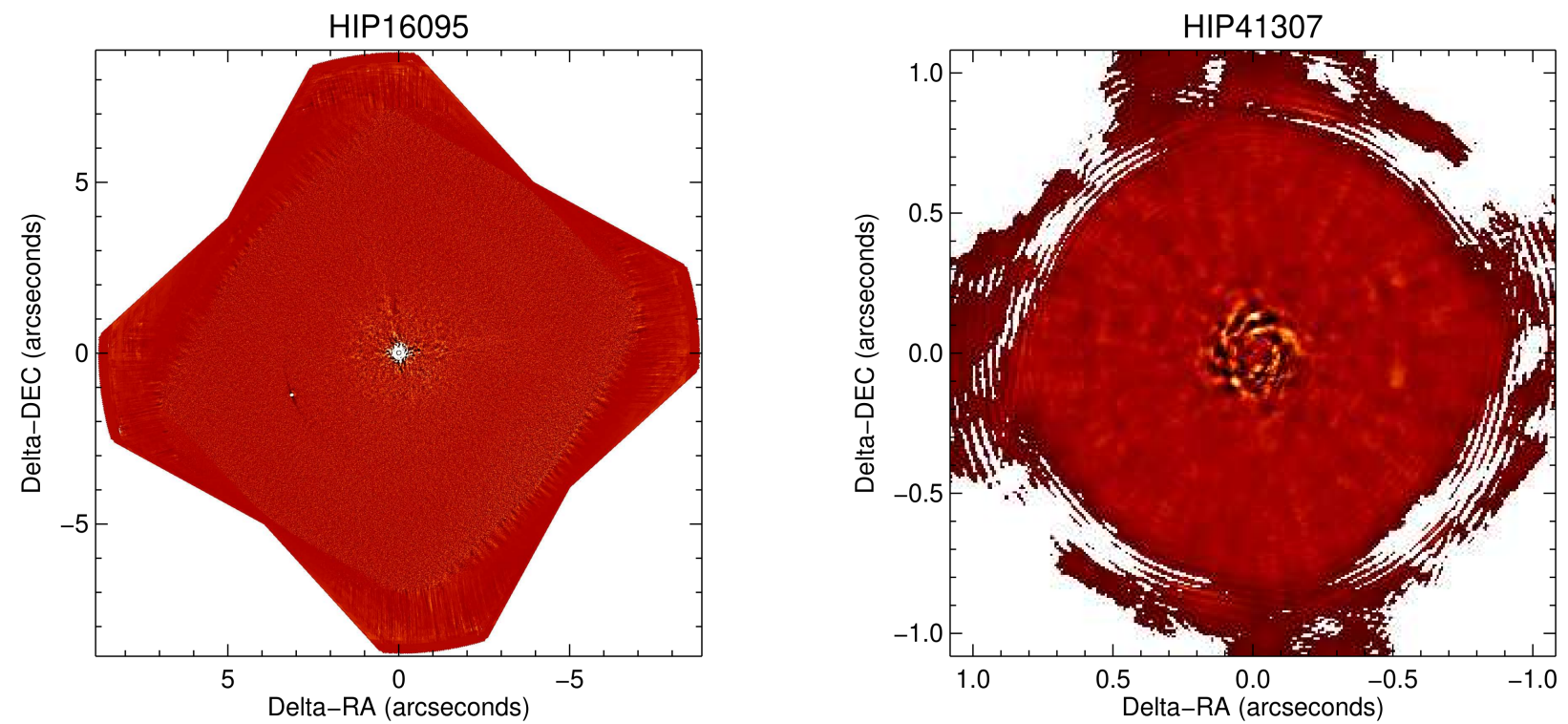

Fig. 3. Left: IRDIS reduced full-frame image of HIP 16095 in the $K_{1}$ and $K_{2}$ combined filters using SpeCal with the TLOCI algorithm (Galicher et al. 2011). A bright companion candidate is well identified at a few arcseconds to the east of the star. North is up, and east is left. Right: IFS image reduced in PCA ASDI of HIP 41307.

the NICI campaign concerning debris disk stars. Both companion candidates around HIP 117452 were earlier identified by De Rosa et al. (2011) in the course of the Volume-limited A-Star (VAST) survey as a candidate binary companion. They were later confirmed by Matthews et al. (2018) as physically bound, confirming that this system was actually a quadruple system with an A0 primary (HIP 117452 A), orbited by a close binary pair Ba and $\mathrm{Bb}$ also resolved in this survey, and additionally by a K-type star at about $75^{\prime \prime}$.

Follow-up observations of the candidates were automatically scheduled and obtained using the DBI mode with $J_{2} J_{3}$ filters of IRDIS, which is well adapted to distinguish background stars from physically young, early-T, or warm mid-L dwarf planets, and offers an additional epoch for a proper motion test.
Follow-up observations were then processed using SpeCal with the TLOCI algorithm in ADI only and a template approach as before. All companion candidates were re-detected, except the one around HIP 8832 falling outside the IRDIS field, given its large separation and an observing sequence that was not perfectly centered with the meridian passage. The results are reported in Table 3. The use of a different pair of filters enabled us to explore the companion candidate photometric properties in the $J_{2}$-band- and $J_{3}$-band-based CMD, for which we also report the distribution of background stars observed in previous crowded fields (see Fig. 4, right). One can directly see that most of our late-L to early-T potential companion candidates, including the previous ones identified as stationary background stars around HIP 34276 (cc1 and cc2) and HIP 101800 (cc1 and cc2), fall onto 
Table 3. Companion candidate characterization and identification.

\begin{tabular}{|c|c|c|c|c|c|c|c|}
\hline Target & UT date & Candidate & Filter & $\begin{array}{l}\text { Separation } \\
\text { (mas) }\end{array}$ & $\begin{array}{l}\text { Position angle } \\
\text { (deg) }\end{array}$ & $\begin{array}{l}\Delta_{\text {Filter-1 }} \\
\text { (mag) }\end{array}$ & $\begin{array}{l}\Delta_{\text {Filter-2 }} \\
\text { (mag) }\end{array}$ \\
\hline \multirow[t]{2}{*}{ HIP 16095} & 57669.2937186 & cc-1 & DK12 & $3368 \pm 2$ & $111.38 \pm 0.04$ & $11.46 \pm 0.12$ & $11.28 \pm 0.12$ \\
\hline & 58092.1556576 & cc-1 & DJ23 & $3385 \pm 2$ & $111.21 \pm 0.02$ & $12.88 \pm 0.08$ & $12.55 \pm 0.09$ \\
\hline \multirow[t]{2}{*}{ HIP 95619} & 57965.1627630 & cc-1 & DK12 & $4564 \pm 3$ & $254.25 \pm 0.03$ & $11.11 \pm 0.51$ & $10.94 \pm 0.54$ \\
\hline & 58613.3454076 & cc-1 & $\mathrm{DJ} 23$ & $4597 \pm 2$ & $255.23 \pm 0.01$ & $12.17 \pm 0.24$ & $11.83 \pm 0.29$ \\
\hline \multirow[t]{3}{*}{ HIP 101800} & 57940.3125070 & $\mathrm{cc}-1$ & DK12 & $4513 \pm 4$ & $89.84 \pm 0.037$ & $12.42 \pm 0.12$ & - \\
\hline & 58653.3759935 & $\mathrm{cc}-1$ & DJ23 & $4418 \pm 2$ & $89.82 \pm 0.01$ & $13.34 \pm 0.17$ & $13.07 \pm 0.15$ \\
\hline & 58653.3759935 & cc-2 & DJ23 & $4021 \pm 2$ & $89.83 \pm 0.01$ & $14.42 \pm 0.19$ & $14.17 \pm 0.17$ \\
\hline \multirow[t]{4}{*}{ HIP 34276} & 57736.2557381 & cc-1 & DK12 & $3108 \pm 7$ & $132.55 \pm 0.11$ & $12.90 \pm 0.12$ & $12.72 \pm 0.13$ \\
\hline & 57736.2557381 & $\mathrm{cc}-2$ & DK12 & $4407 \pm 4$ & $138.56 \pm 0.06$ & $12.26 \pm 0.12$ & $12.30 \pm 0.12$ \\
\hline & 58445.3349875 & $\mathrm{cc}-1$ & DJ23 & $3124 \pm 4$ & $133.01 \pm 0.06$ & $14.58 \pm 0.29$ & $14.34 \pm 0.12$ \\
\hline & 58445.3349875 & $\mathrm{cc}-2$ & DJ23 & $4421 \pm 5$ & $138.95 \pm 0.06$ & $14.30 \pm 0.29$ & $14.02 \pm 0.13$ \\
\hline \multirow[t]{2}{*}{ HIP 117452} & 57949.3975893 & $\mathrm{Ba}$ & DK12 & $3708 \pm 9$ & $238.09 \pm 0.15$ & $3.84 \pm 0.05$ & $3.76 \pm 0.05$ \\
\hline & & $\mathrm{Bb}$ & DK12 & & & $4.58 \pm 0.05$ & $4.51 \pm 0.05$ \\
\hline HIP 8832 & 57974.3996411 & $\mathrm{cc}-1$ & DK12 & $5674 \pm 3$ & $213.71 \pm 0.04$ & $11.47 \pm 0.50$ & $11.54 \pm 0.51$ \\
\hline
\end{tabular}

Notes. Target name and observing date (modified Julian day) are given, as well as the different sources identified with their relative position and relative flux.
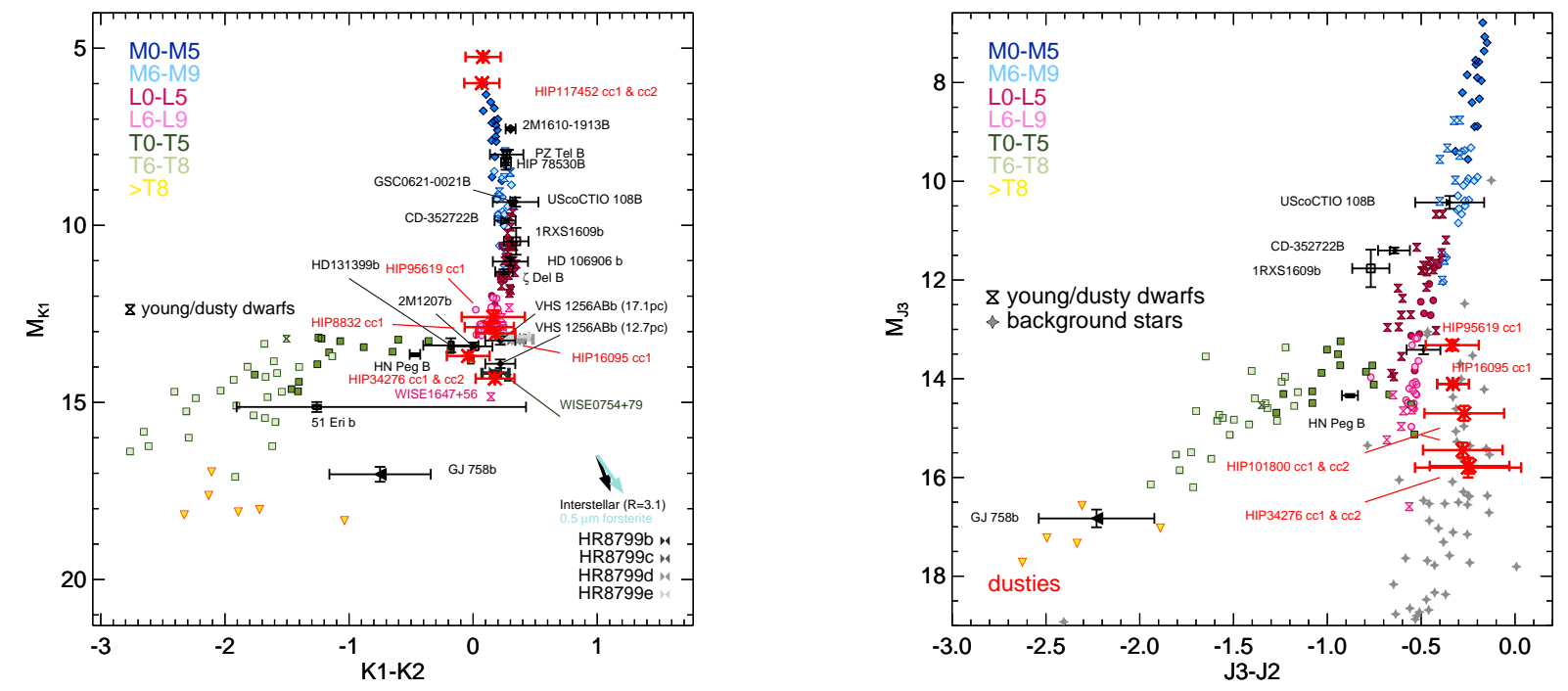

Fig. 4. Left: absolute magnitude in $K_{1}$-band versus $K_{1}-K_{2}$ color for brown dwarfs with discovered companions. Right: same, but for absolute magnitude in $J_{3}$-band versus $J_{3}-J_{2}$ color. The targets from our survey are noted in red.

the background contaminant sequence indicating that they are most likely background stars. As a further check, we used the relative astrometry obtained at two epochs to estimate the proper motion of the companion candidates relative to their primary stars. Figure 5 shows the proper motion plots of each candidate and confirms that the companion candidates around HIP 34276, HIP 101800, and HIP 95619 are not co-moving with their primary stars. The distance and proper motion of the stars, with their uncertainties, are taken from the Gaia Data Release 2 catalog (Gaia Collaboration 2018). For HIP 16095, given the relatively low proper motion of the star, the status of the companion candidate HIP 16095-cc1 remains ambiguous. However, the $J_{2}$-bandand $J_{3}$-band-based CMD still supports a background contamination. If bound, this candidate would have an estimated mass between 7 and $12 M_{\text {Jup }}$ at the system age ( $\left.\leq 100 \mathrm{Myr}\right)$ and distance $(88 \mathrm{pc})$ illustrative of the SPHERE detection performances around young nearby stars beyond $10 \mathrm{au}$.

For HIP $117452 \mathrm{Ba}$ and $\mathrm{Bb}$, the colors and magnitudes in $K_{1}$ and $K_{2}$ compared to the predictions of the evolutionary models of Siess et al. (2000) suggest that $\mathrm{Ba}$ and $\mathrm{Bb}$ are a pair of M1 and M2 low-mass stars, considering an age of $40 \mathrm{Myr}$ at a distance of $42 \mathrm{pc}$. Combining our relative astrometry with the one reported by Matthews et al. (2018) and shown in Table 3, we performed a first orbit fitting of the pair. Following the method developed by Chauvin et al. (2012), we used a Markov chain Monte Carlo (MCMC) Bayesian analysis technique (Ford \& Gregory 2007), which is well suited for observations covering a small part of the whole orbit (for large orbital periods). We did not consider any prior information using the proximity of the primary star. The results are reported in Fig. A.1 and favor a relatively inclined orbit $i \sim 98_{-5}^{+8} \mathrm{deg}$, a longitude of ascending node fairly well-constrained at $\Omega=20 \pm 2 \mathrm{deg}$, tight semi-major axis $a \sim 14_{-4}^{+7}$ au, but surprisingly large eccentricities $e \geq 0.4$. These large values of eccentricity are not dynamically expected, given the proximity of the primary star located at a physical projected separation of $\sim 150$ au, although the orbit of the binary companion around HIP 117452 is not known. Fitting solutions using a least squares Levenberg-Marquardt (LSLM) algorithm 

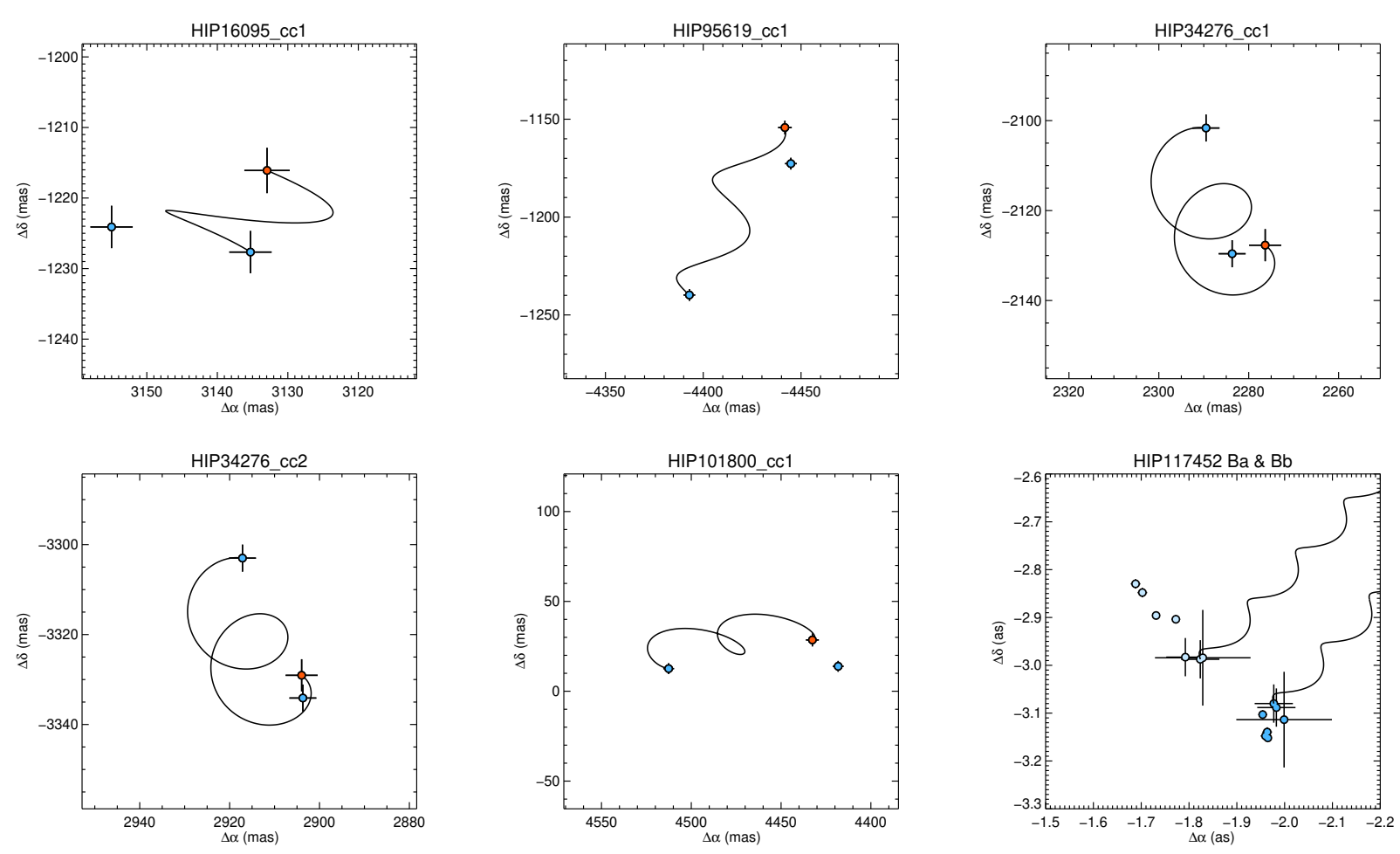

Fig. 5. SPHERE measurements (in blue) of the offset positions of the companion candidates relative to their primary stars. For each diagram, if the candidate is a stationary background object, the expected variation of offset positions is shown (solid line). This is based on a distance and on a primary proper motion, as well as the initial offset position of the candidate relative to the primary. The predicted offset positions of a stationary background object for the second epoch is shown in red with uncertainties. For HIP117452, measurements of both components Ba and Bb at various epochs are plotted in dark and light blue, respectively.

(Press et al. 1992) to search for the model with the minimal reduced $\chi^{2}$ are also reported for comparison. Further dynamical study of the global system considering the debris disk architecture around HIP 117452 and the binary companion HIP $117452 \mathrm{BaBb}$ configuration is be needed.

\section{Detection limits and survey completeness}

To exploit the information from the actual nondetection in IFS and IRDIS observations of the survey, the detection limits of each individual observations were then estimated. Based on SpeCal results, we derived a standard pixel-to-pixel noise map for each observing sequence corrected from the flux loss related to the ADI or ASDI processing by injecting fake planets. The detection limit maps at $5 \sigma$ were then obtained using the pixel-topixel noise map divided by the flux loss and normalized by the relative calibration with the primary star (considering the different exposure times, the neutral density, and the coronograph transmission). These detection limits were finally corrected from small number statistics following the prescription of Mawet et al. (2014) to adapt our $5 \sigma$ confidence level at small angles with IRDIS and IFS. The $5 \sigma$ contrast curves, resulting from the azimuthal average of the detection maps, are reported for IFS and IRDIS in Fig. 6.

To convert the detection limits in terms of the mass and semimajor axis parameter space explored with SPHERE, we used the multi-purpose exoplanet simulation system (MESS) code, a Monte Carlo tool for the statistical analysis and prediction of exoplanet search results (Bonavita et al. 2012). This code has been used extensively in previous direct imaging surveys for that same purpose (Chauvin et al. 2010, 2015, 2018; Vigan et al. 2012, 2017; Rameau et al. 2013; Lannier et al. 2016). With MESS, we then generated a uniform grid of mass and semi-major axis in the interval $[1,80] M_{\mathrm{Jup}}$ and $[1,1000]$ au with a sampling of $0.5 M_{\mathrm{Jup}}$ and $1 \mathrm{au}$, respectively.

For each point in the grids, 100 orbits were generated, randomly oriented in space from uniform distributions in $\cos (i)$, $\omega, \Omega, e \leq 0.8$, and $T_{p}$. We built detection probability maps by counting the number of detected planets over the number of generated ones and simply comparing the on-sky projected position (separation and position angle) of each synthetic planet with the SPHERE 2D detection limit maps at $5 \sigma$ converted in masses based on the COND (hot-start) model predictions (Baraffe et al. 2003). The primary age, distance, and magnitude reported in Table 1 are considered for the luminosity-mass conversion.

The resulting detection probability map of the complete survey is reported in Fig. 7. This result shows that, despite the relatively wide age range (20-120 Myr) and distance (10-102 pc) of our sample, we achieved a relatively good detection probability larger than $50 \%$ for giant planets with masses larger than $5 M_{\text {Jup }}$ and semi-major axes between 10 and $500 \mathrm{au}$, sufficient for the detection of system analogs to HR 8799 or HD 95086. In principle, the degeneracy between mass and initial entropy could change these limits considerably (e.g., Marleau \& Cumming 2014; Brandt et al. 2014). In practice, however, taking more realistic post-formation entropies into account strongly mitigates this problem, as shown for instance in the case of HIP 65426 b by Marleau et al. (2019).

\section{Discussion}

Our survey is composed of relatively old gas-free systems. Therefore, some of these systems contain debris disks. We assumed that planets are a valid explanation for the formation of 

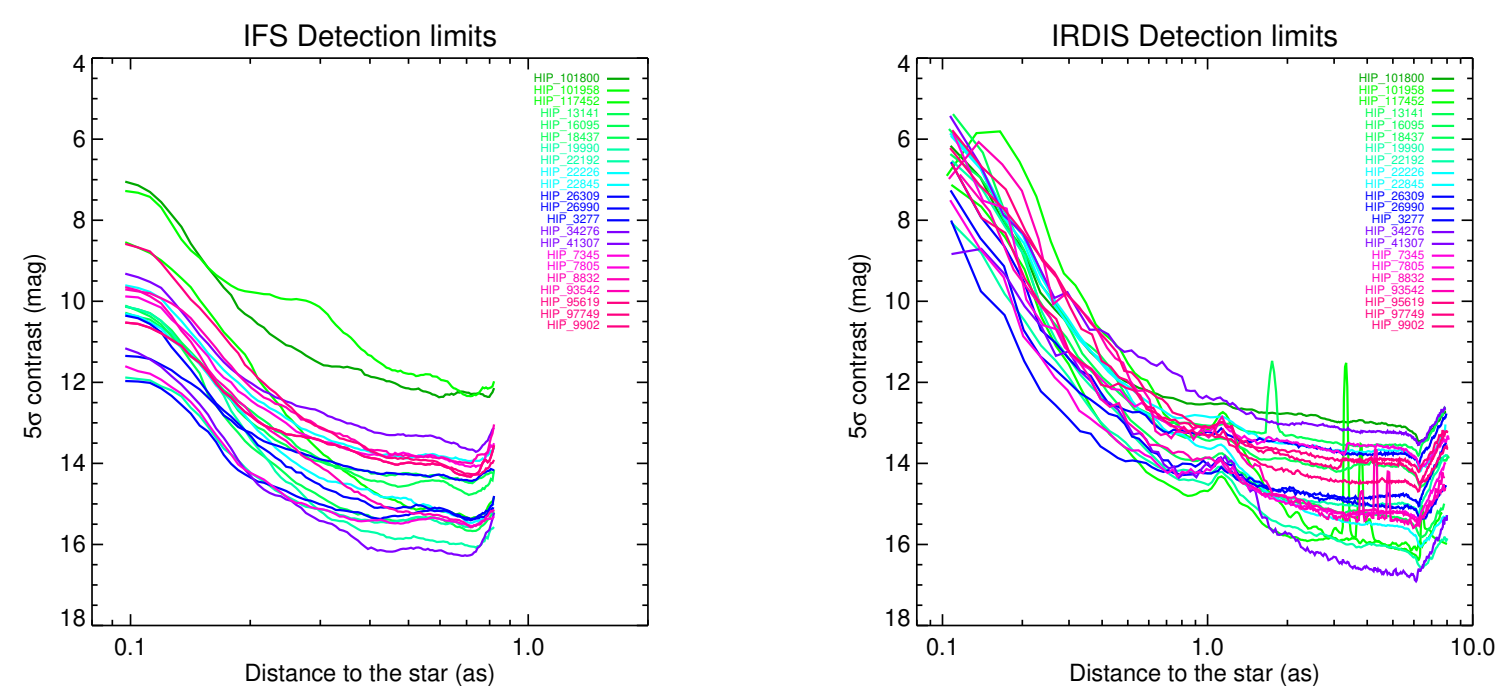

Fig. 6. Magnitude contrast limit curves for all targets with TLOCI algorithm.

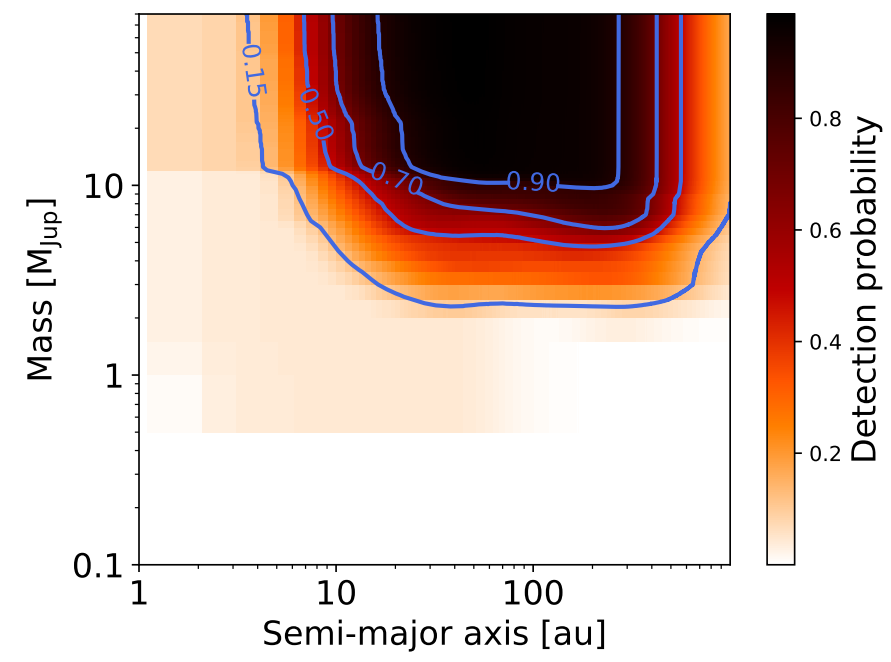

Fig. 7. Combined mean detection probability map for the whole survey.

debris structure, as shown in the case of the Solar System where planets are known to reside between two belts of debris, and in the case of HR 8799 and HD 95086 where planets are known to reside in two-temperature debris disks. The analysis of our survey follows the work by Matthews et al. (2018). The temperature values of debris belts are found in Chen et al. (2014), where these temperatures were estimated using a two-temperature black-body model and a Bayesian parameter estimation to select the best model to fit the SED. The disk radii were calculated following Pawellek \& Krivov (2015), assuming that dust are composed of $50 \%$ astrosilicate and $50 \%$ ice. In addition, we constrained our SPHERE/IRDIS observations with dynamical arguments on the possible planetary systems hiding within the debris gaps (Shannon et al. 2016).

Mass limits were calculated with the MESS code as described in Sect. 6 and shown in Fig. 8. The theoretical mass for a single planet to clear the observed gap is large $\geq 25 M_{\mathrm{J}}$ (Nesvold \& Kuchner 2014). Therefore, in our cases, we infer that the systems must be in multi-planet configuration, as in HR 8799, in which several planets with lower masses clear the gap. In Fig. 8, we plot the minimum masses of planets required to clear the debris gaps, as well as their location and their "Np" number based on the $N$-body simulations of Shannon et al. (2016). This model considers only planets with low eccentricities. The mass and the $\mathrm{Np}$ number change if the eccentricity is larger. The mass, shown in green in Fig. 8, is the minimum mass per planet, with uncertainties based on the age of the system and on the belts radius. The minimum mass calculation assumes that planets are spaced by a typical value of $\sim 20$ mutual Hill radius $\left(R_{\mathrm{H}}\right)$, which is consistent with the value of $21.7 \pm 9.5 R_{\mathrm{H}}$ predicted by Fang \& Margot (2013).

By combining the observational upper and theoretical lower mass constrains, only a small region of parameter space is unconstrained. For 12 targets in our survey of which the temperature values are found in Chen et al. (2014), we infer a multi-planet system based on the large theoretical clearing masses. In such a multi-planet system, the widest separation planet would have a physical separation close to that of the outer debris belt, where our direct imaging limits are relatively tight. In main cases, planets must be at least $\sim 0.1 M_{\mathrm{J}}$ to clear the observed gap based on dynamical arguments, and in some cases the dynamical mass limit exceeds $1 M_{\mathrm{J}}$. In Fig. 8, for the target HIP 7345, the mass limit, $\sim 1.3 M_{\mathrm{J}}$ at $90 \%$ in the gap, is close to the dynamical mass limit $\sim 0.9$.

Among our 12 targets for which we note the presence of two debris belts, no exoplanetary mass companions were detected. Our sample is too small for a detailed statistical analysis. However, a nondetection in a sample of 12 stars is not inconsistent with the debris disk occurrence rate of $6.27 \%$ in a debris disk sample of planets between 5-20 $M_{\mathrm{J}}$ and 10-1000 au (Meshkat et al. 2017), since we would expect that some companions might be below our detection limits. Our nondetections are also consistent with the lower occurrence rate of $\sim 1 \%$ found in Bowler (2016) and Galicher et al. (2016). The results of this 12 target sample are not incompatible with the theory that planets are carving wide debris gaps, since in each case our direct imaging mass limits are higher than the theoretical mass limits that we calculate.

The existence of the planetary perturbers beyond $5 \mathrm{au}$, and potentially these architectures will be explored in futur observations: (i) observations combining radial velocity, astrometry with Gaia for the inner parts ( $\leq 5 \mathrm{au}$ ), (ii) observations with the next generation of planet imagers from the ground (SCExAO, KPIC, SPHERE+, GPI2.0 on 10m-class Telescopes, then with the ELTs) and space (JWST, WFIRST). 


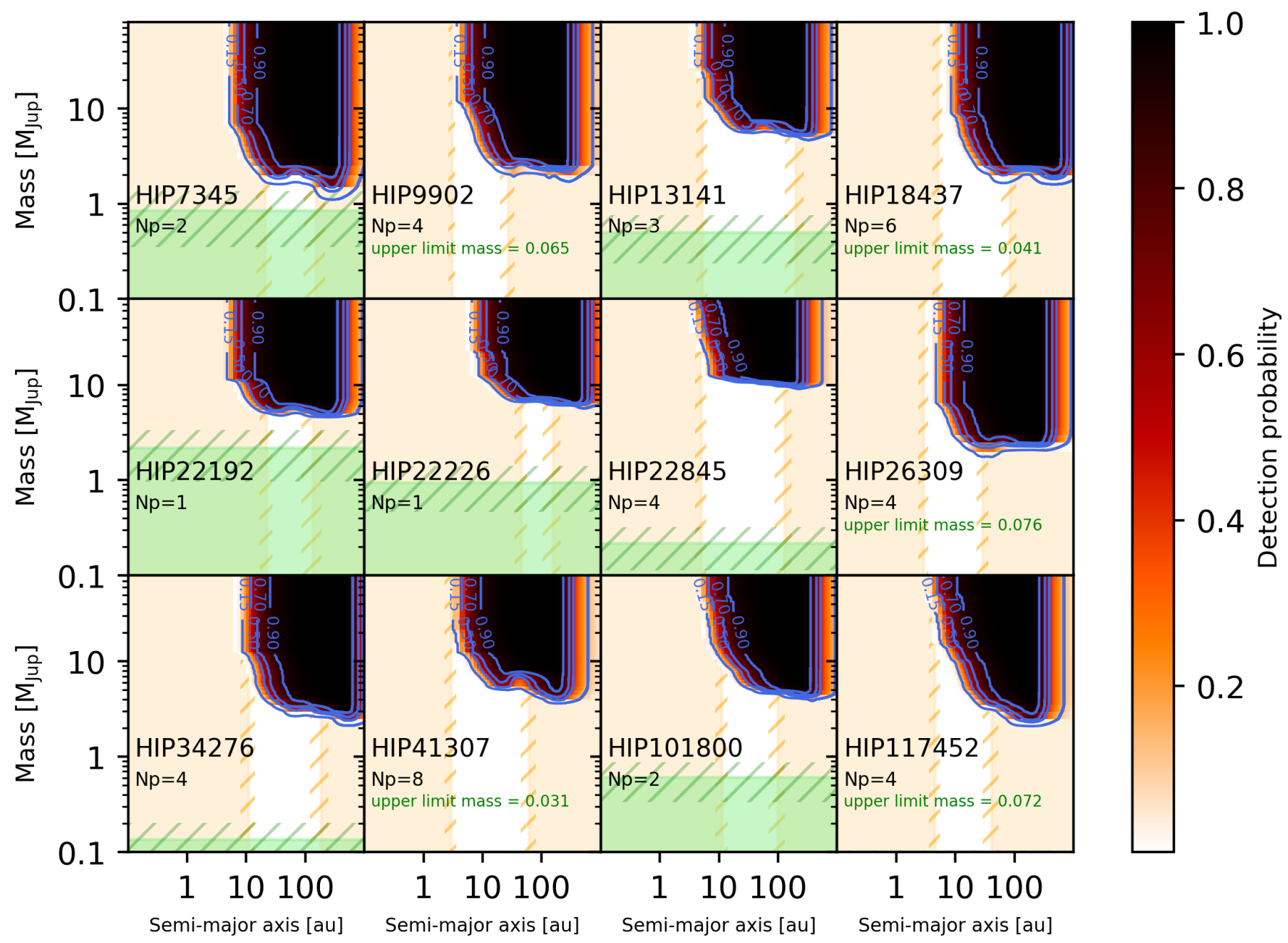

Fig. 8. Constraints on planetary systems for 12 targets in our survey. The positions of the inner and the outer debris belts are shown in orange by shading the regions inside the inner and beyond the outer. Our mass contrast limits are based on SPHERE/IRDIS and COND model predictions. Dynamical mass constraints for a slightly closer planet spacing of 20 mutual Hill radii from Shannon et al. (2016) are shown in green with masses below this value shaded. $\mathrm{Np}$ is the number of planets with the mass (indicated in green) required to open the gap. The uncertainties for debris belt position and dynamical mass limit are calculated based on the uncertainty in debris belt temperature, and indicated with hatching.

\section{Conclusions}

We reported the observations and analysis of a survey of 22 stars with VLT/SPHERE with IRDIS in the DBI mode with $K_{1} K_{2}$ filters and $J_{2} J_{3}$ for the follow-up observations, and IFS in the $Y-H$ filters, with the goal of detecting and characterizing giant planets on wide orbits. The selected sample favors young, that is to say $\leq 100 \mathrm{Myr}$, nearby, $\leq 100 \mathrm{pc}$, dusty, and early-type stars to maximize the range of mass and separation, over which the observations are sensitive. The optimized observation strategy with the angular differential imaging in thermal bands and a dedicated data reduction using various algorithms allow us to reach a typical contrast $12.5 \mathrm{mag}$ at $0.25^{\prime \prime}$ and $14 \mathrm{mag}$ at $1.0^{\prime \prime}$ in IRDIS. These contrasts are converted to mass limits for each target. Despite the good sensitivity of our survey, we did not detect any new giant planets. We confirmed that the sources detected around HIP 34276, HIP 101800, HIP 16095, and HIP 95619 are stationary background sources by analyzing $K_{1}$-band, $K_{2}$-band, $J_{2}$-band, and $J_{3}$-band images and their relative motions. The status of the candidate around HIP 8832 still requires further follow-up. HIP $117452 \mathrm{BaBb}$ is resolved and confirmed as a binary companion (De Rosa et al. 2011; Matthews et al. 2018). For 12 targets of our survey, where we determined the radii of the debris belt, we derived upper and lower mass limits. We used Monte Carlo simulations to estimate the sensitivity survey performance in terms of planetary mass and semi-major axis to perform the upper limit. We additionally calculated the minimum required mass for planets in the system to have cleared the observed debris gap to perform the lower mass limit. Combining our upper and lower mass limits, we are able to tightly constrain the unexplored parameter space around these systems: typically, planets must be at least $\sim 0.1 M_{\mathrm{J}}$ in main cases to clear the observed gap based on dynamical arguments, and in some cases the dynamical limit exceeds $1 M_{\mathrm{J}}$. Direct imaging data from VLT/SPHERE are sensitive to planets of $\sim 3 M_{\mathrm{J}}$ for a typical target in our survey. Several of the planetary systems will likely be detectable with the next generation of high-contrast imagers.

Acknowledgements. First, we thank the referee for providing useful comments. This project was partly supported by the IDEXLyon project (contract ANR16-IDEX-0005) under the auspices University of Lyon. It was supported by CNRS, by the Agence Nationale de la Recherche (ANR-14-CE33-0018). It has received funding from the European Union's Horizon 2020 research and innovation programme under the Marie Skłodowska-Curie grant agreement No 823823. G.-D. Marleau acknowledges the support of the DFG priority program SPP 1992 "Exploring the Diversity of Extrasolar Planets" (KU 2849/7-1). C.M. and G.D.M. acknowledge support from the Swiss National Science Foundation under 
grant BSSGI0_155816 "PlanetsInTime”. Parts of this work have been carried out within the frame of the National Centre for Competence in Research PlanetS supported by the SNSF. A.B. acknowledges support from ICM (Iniciativa Científica Milenio) via the Núcleo Milenio de Formación Planetaria, and from FONDECYT (grant 1190748). Finally, this work has made use of the SPHERE Data Centre, jointly operated by OSUG/IPAG (Grenoble), PYTHEAS/LAM/CESAM (Marseille), OCA/Lagrange (Nice), Observatoire de Paris/LESIA (Paris), and Observatoire de Lyon, also supported by a grant from Labex OSUG@2020 (Investissements d'avenir - ANR10 LABX56).

\section{References}

Baraffe, I., Chabrier, G., Barman, T. S., Allard, F., \& Hauschildt, P. H. 2003, A\&A, 402, 701

Bell, C. P., Mamajek, E. E., \& Naylor, T. 2015, MNRAS, 454, 593

Beuzit, J.-L., Vigan, A., Mouillet, D., et al. 2019, A\&A, 631, A155

Boccaletti, A., Abe, L., Baudrand, J., et al. 2008, Proc. SPIE, 7015, 70151B

Boley, A. C. 2009, ApJ, 695, L53

Bonavita, M., Chauvin, G., Desidera, S., et al. 2012, A\&A, 537, A67

Bonnefoy, M., Perraut, K., Lagrange, A.-M., et al. 2018, A\&A, 618, A63

Bowler, B. P. 2016, PASP, 128, 102001

Brandt, T. D., McElwain, M. W., Turner, E. L., et al. 2014, ApJ, 794, 159

Bromley, B. C., \& Kenyon, S. J. 2014, ApJ, 796, 141

Cameron, A. 1978, Moon Planets, 18, 5

Cantalloube, F., Mouillet, D., Mugnier, L., et al. 2015, A\&A, 582, A89

Chauvin, G., Lagrange, A. M., Bonavita, M., et al. 2010, A\&A, 509, A52

Chauvin, G., Lagrange, A.-M., Beust, H., et al. 2012, A\&A, 542, A41

Chauvin, G., Vigan, A., Bonnefoy, M., et al. 2015, A\&A, 573, A127

Chauvin, G., Gratton, R., Bonnefoy, M., et al. 2018, A\&A, 617, A76

Chen, C. H., Mittal, T., Kuchner, M., et al. 2014, ApJS, 211, 25

Claudi, R. U., Turatto, M., Gratton, R. G., et al. 2008, Proc. SPIE, 7014, 70143E

Crida, A., Masset, F., \& Morbidelli, A. 2009, ApJ, 705, L148

David, T. J., \& Hillenbrand, L. A. 2015, ApJ, 804, 146

De Rosa, R. J., Bulger, J., Patience, J., et al. 2011, MNRAS, 415, 854

De Rosa, R. J., Patience, J., Ward-Duong, K., et al. 2014, MNRAS, 445, 3694

Delorme, P., Meunier, N., Albert, D., et al. 2017, in SF2A-2017: Proceedings of the Annual meeting of the French Society of Astronomy and Astrophysics, 347

Dipierro, G., Price, D., Laibe, G., et al. 2015, MNRAS, 453, L73

Dohlen, K., Langlois, M., Saisse, M., et al. 2008, Proc. SPIE, 7014, 70143L

Fang, J., \& Margot, J.-L. 2013, ApJ, 767, 115

Ford, E., \& Gregory, P. 2007, in Statistical Challenges in Modern Astronomy IV (San Francisco: ASP), 371, 189

Fusco, T., Rousset, G., Sauvage, J.-F., et al. 2006, Opt. Express, 14, 7515

Gaia Collaboration (Brown, A. G. A., et al.) 2018, A\&A, 616, A1

Galicher, R., Marois, C., Macintosh, B., Barman, T., \& Konopacky, Q. 2011, ApJ, 739, L41

Galicher, R., Marois, C., Macintosh, B., et al. 2016, A\&A, 594, A63

Galicher, R., Boccaletti, A., Mesa, D., et al. 2018, A\&A, 615, A92

Gauza, B., Béjar, V. J. S., Pérez-Garrido, A., et al. 2015, ApJ, 804, 96

Greco, J. P., \& Brandt, T. D. 2016, ApJ, 833, 134

Hugot, E., Ferrari, M., El Hadi, K., et al. 2012, A\&A, 538, A139

Kalas, P., Graham, J. R., Chiang, E., et al. 2008, Science, 322, 1345

Kennedy, G. M., \& Kenyon, S. J. 2008, ApJ, 682, 1264

Kennedy, G. M., \& Wyatt, M. C. 2014, MNRAS, 444, 3164

Lafrenière, D., Marois, C., Doyon, R., Nadeau, D., \& Artigau, É. 2007, ApJ, 660 770

Lagrange, A.-M., Gratadour, D., Chauvin, G., et al. 2009, A\&A, 493, L21

Lagrange, A.-M., Bonnefoy, M., Chauvin, G., et al. 2010, Science, 329, 57
Lagrange, A.-M., Boccaletti, A., Milli, J., et al. 2012, A\&A, 542, A40 Lambrechts, M., \& Johansen, A. 2012, A\&A, 544, A32

Lannier, J., Delorme, P., Lagrange, A. M., et al. 2016, A\&A, 596, A83 Macintosh, B., Graham, J. R., Barman, T., et al. 2015, Science, 350, 64

Maire, A.-L., Langlois, M., Dohlen, K., et al. 2016, Proc. SPIE, 9908, 990834

Marleau, G.-D., \& Cumming, A. 2014, MNRAS, 437, 1378

Marleau, G.-D., Coleman, G. A. L., Leleu, A., \& Mordasini, C. 2019, A\&A, 624 A20

Marois, C., Lafrenière, D., Doyon, R., Macintosh, B., \& Nadeau, D. 2006, ApJ, 641, 556

Marois, C., Zuckerman, B., Konopacky, Q. M., Macintosh, B., \& Barman, T. 2010, Nature, 468, 1080

Marois, C., Correia, C., Galicher, R., et al. 2014, SPIE, 9148, 91480U

Matthews, B. C., Krivov, A. V., Wyatt, M. C., Bryden, G., \& Eiroa, C. 2014, in Protostars and Planets VI, eds. H. Beuther, R. S. Klessen, C. P. Dullemond, $\&$ T. Henning (Tucson, AZ: University of Arizona Press), 521

Matthews, E., Hinkley, S., Vigan, A., et al. 2018, MNRAS, 480, 2757

Mawet, D., Milli, J., Wahhaj, Z., et al. 2014, ApJ, 792, 97

Mayor, M., Queloz, D., Marcy, G., et al. 1995, IAU Circ., 6251

Mesa, D., Gratton, R., Zurlo, A., et al. 2015, A\&A, 576, A121

Mesa, D., Vigan, A., D’Orazi, V., et al. 2016, A\&A, 593, A119

Meshkat, T., Mawet, D., Bryan, M. L., et al. 2017, AJ, 154, 255

Moór, A., Kóspál, Á., Ábrahám, P., et al. 2016, ApJ, 826, 123

Mouillet, D., Larwood, J. D., Papaloizou, J. C. B., \& Lagrange, A. M. 1997, MNRAS, 292, 896

Nesvold, E. R., \& Kuchner, M. J. 2014, ApJ, 798, 83

Pavlov, A., Möller-Nilsson, O., Feldt, M., et al. 2008, Proc. SPIE, 7019, 701939

Pawellek, N., \& Krivov, A. V. 2015, MNRAS, 454, 3207

Petit, C., Sauvage, J.-F., Fusco, T., et al. 2014, Proc. SPIE, 9148, 914800

Pinte, C., Price, D., Ménard, F., et al. 2020, ApJ, 890, L9

Pollack, J. B., Hubickyj, O., Bodenheimer, P., et al. 1996, Icarus, 124, 62

Press, W. H., Teukolsky, S. A., Vetterling, W. T., \& Flannery, B. P. 1992, Numerical recipes in FORTRAN. The Art of Scientific Computing (Cambridge: Cambridge University Press)

Racine, R., Walker, G. A. H., Nadeau, D., Doyon, R., \& Marois, C. 1999, PASP, 111,587

Rameau, J., Chauvin, G., Lagrange, A. M., et al. 2013, A\&A, 553, A60

Rhee, J. H., Song, I., Zuckerman, B., \& McElwain, M. 2007, ApJ, 660, 1556

Samland, M., Mollière, P., Bonnefoy, M., et al. 2017, A\&A, 603, A57

Sauvage, J.-F., Fusco, T., Petit, C., et al. 2010, Proc. SPIE, 7736, 77360F

Schneider, G., Grady, C. A., Hines, D. C., et al. 2014, AJ, 148, 59

Shannon, A., Bonsor, A., Kral, Q., \& Matthews, E. 2016, MNRAS, 462, L116

Siess, L., Dufour, E., \& Forestini, M. 2000, A\&A, 358, 593

Soummer, R., Aime, C., Ferrari, A., et al. 2005, Proc. Int. Astron. Union, 1, 367

Soummer, R., Pueyo, L., \& Larkin, J. 2012, ApJ, 755, L28

Stone, J. M., Skemer, A. J., Kratter, K. M., et al. 2016, ApJ, 818, L12

Su, K. Y., Morrison, S., Malhotra, R., et al. 2015, ApJ, 799, 146

Thalmann, C., Schmid, H. M., Boccaletti, A., et al. 2008, Proc. SPIE, 7014 70143F

Vigan, A., Moutou, C., Langlois, M., et al. 2010, MNRAS, 407, 71

Vigan, A., Patience, J., Marois, C., et al. 2012, A\&A, 544, A9

Vigan, A., Bonavita, M., Biller, B., et al. 2017, A\&A, 603, A3

Wahhaj, Z., Liu, M. C., Nielsen, E. L., et al. 2013, ApJ, 773, 179

Zuckerman, B. 2001, ARA\&A, 39, 549

Zuckerman, B., \& Song, I. 2004a, ARA\&A, 42, 685

Zuckerman, B., \& Song, I. 2004b, ApJ, 603, 738

Zuckerman, B., \& Song, I. 2012, ApJ, 758, 77

Zuckerman, B., Forveille, T., \& Kastner, J. H. 1995, Nature, 373, 494

Zuckerman, B., Rhee, J. H., Song, I., \& Bessell, M. 2011, ApJ, 732, 61

Zuckerman, B., Vican, L., Song, I., \& Schneider, A. 2013, ApJ, 778, 5 


\section{Appendix A: MCMC orbital fit of HIP $117452 \mathrm{BaBb}$}

a (AU)
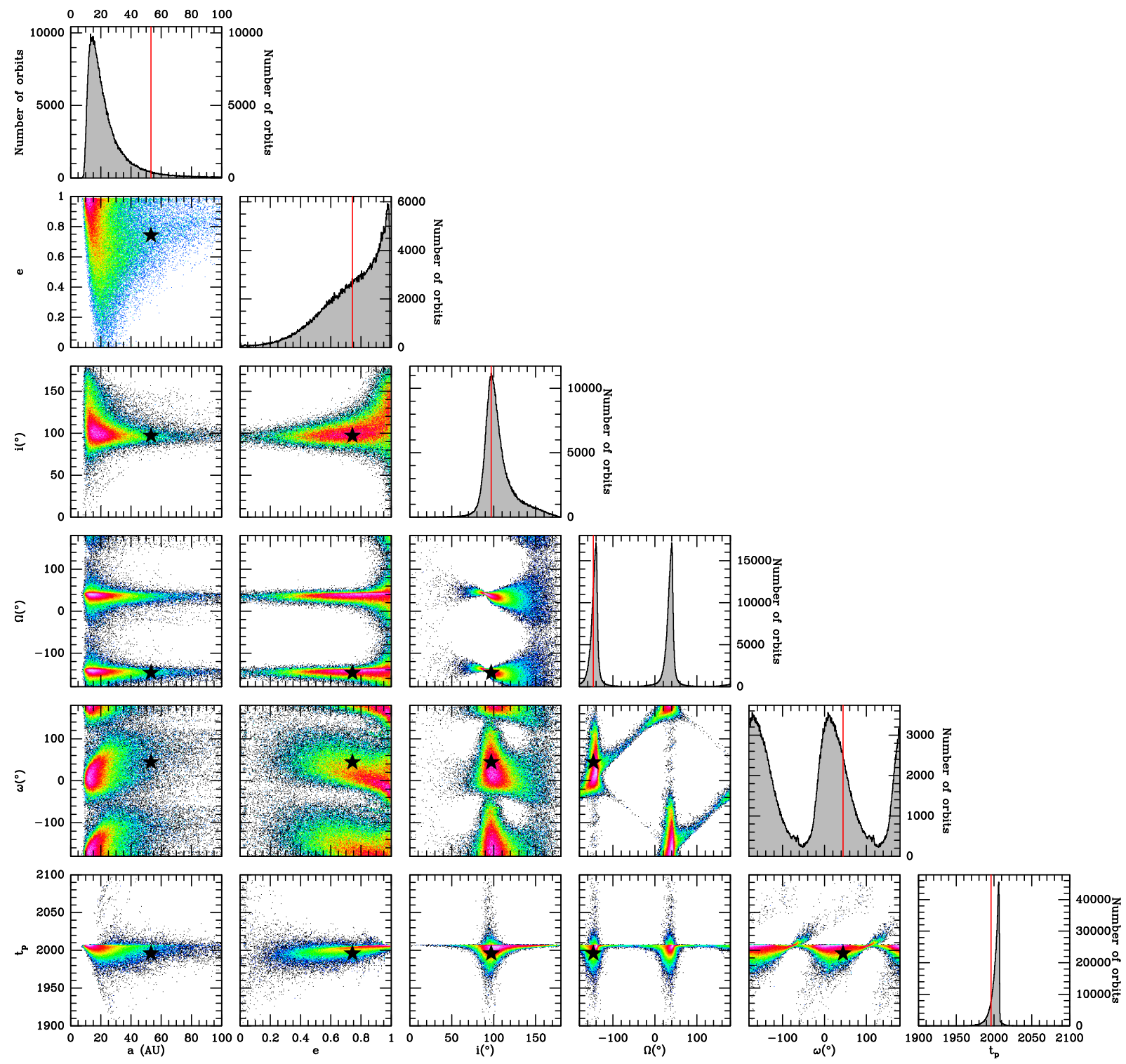

Fig. A.1. Results of the MCMC fit of the NaCo and SPHERE combined astrometric data of HIP $117452 \mathrm{Ba}$ and Bb reported in terms of statistical distribution matrix of the orbital elements $a, e, i, \Omega, \omega$, and $t_{p}$. The red line in the histograms and the black star in the correlation plots indicate the position of the best LSLM- $\chi^{2}$ model obtained for comparison. 